\title{
La BD : un support « facilitant » en classe de SES ?
}

\section{Claire Polo et Nicolas Rouvière}

\section{OpenEdition}

\section{Journals}

Édition électronique

URL : http://journals.openedition.org/trema/5003

DOI : $10.4000 /$ trema.5003

ISSN : 2107-0997

\section{Éditeur}

Faculté d'Éducation de l'université de Montpellier

Édition imprimée

ISBN : 979-10-96627-07-3

ISSN : 1167-315X

\section{Référence électronique}

Claire Polo et Nicolas Rouvière, « La BD : un support « facilitant » en classe de SES ? », Tréma [En ligne], 51 | 2019, mis en ligne le 01 avril 2019, consulté le 06 août 2019. URL : http://journals.openedition.org/ trema/5003 ; DOl : 10.4000/trema.5003

Ce document a été généré automatiquement le 6 août 2019

Trema 


\title{
La BD : un support « facilitant » en classe de SES?
}

\author{
Claire Polo et Nicolas Rouvière
}

\section{Introduction}

1 La classe de sciences économiques et sociales (SES) constitue typiquement un espace didactique où la bande dessinée (BD) est utilisée comme simple médium, au titre de document qui, comme le texte, ne mériterait pas d'apprentissage spécifique. Le recours à la BD est plutôt perçu comme facilitant en soi l'apprentissage, qu'il s'agisse de motiver les élèves dans leur lecture documentaire, ou de simplifier l'explication de concepts complexes. Nombre de manuels scolaires intègrent des «strips » (BD brèves de quelques cases) parmi les documents de travail proposés. Cependant, le postulat selon lequel le langage du $9 \mathrm{e}$ art serait facilitant en soi et ne nécessiterait pas de médiation spécifique, $a$ fortiori pour des élèves de niveau lycée, est mis à mal par la littérature (Helsby, 1999; Lebrun \& Lacelle, 2010, 2011; Missiou, 2012 ; Lacelle, 2012). Par ailleurs, les enquêtes sur les pratiques culturelles des Français (2008; BPI \& DEPS, 2011) montrent que les plus gros lecteurs de BD sont aussi en général les plus gros lecteurs ${ }^{1}$. On pourrait donc s'attendre à ce que la BD, plutôt que de constituer une vulgarisation attractive et efficace pour toute une classe, maintienne un écart entre les personnes ayant une disposition à lire, et celles qui ne lisent pas ou peu, disposition socialement distribuée, généralement associée au capital culturel des classes moyennes ou supérieures (Evans, 2015, p. 29-31). Sans prétendre épuiser la discussion à propos de ces questions, nous proposons ici l'analyse d'une mise en œuvre a priori problématique au plan didactique, où une BD relativement longue est utilisée en classe de Première SES, comme support d'évaluation écrite, sans travail préalable explicite sur les compétences spécifiques nécessaires à la lecture d'un tel support. L'enseignante est co-auteure de cet article. Elle a pensé que le support BD serait motivant pour ses élèves et que la dimension figurative leur permettrait de se représenter un parcours de vie sous une forme plus incarnée. Cette libre initiative ne s'est pas inscrite dans un protocole expérimental. C'est après-coup, en en discutant avec un collègue, second auteur de cet article, que l'idée d'étudier les effets de ce choix a germé. 
Après avoir précisé la situation didactique au regard des enjeux de savoirs en classe de SES et au regard de la difficulté sémiotique présentée par la lecture du support mobilisé (I), nous nous intéressons à ce qu'a produit l'introduction relativement brutale d'une telle BD pour l'évaluation, en couplant l'analyse des copies d'élèves avec celle de leurs réponses à un questionnaire sur leurs pratiques de lecture et leur perception de la tâche. Les effets de cette pratique didactique ne se révèlent pas globalement univoques (II). Des études de cas montrent que l'image a pu parfois faciliter la compréhension du récit, tout en favorisant à d'autres endroits des raccourcis erronés, avec des impacts divers sur la réussite de la tâche (III). Nous proposons, pour rendre compte de ces résultats, d'introduire la notion de support subjectivement facilitant (IV). Cela nous amène à discuter de la possibilité de mieux rendre compte des situations authentiques en intégrant davantage, dans les modèles didactiques traditionnels, la prise en compte de la subjectivité, et notamment des émotions liées au support didactique.

\section{Une situation de lecture constituant a priori une pratique didactique problématique}

\section{Une pratique en relative rupture avec des principes didactiques fondamentaux}

2 La pratique dont il est question ici se situe à la fin de la séquence d'enseignement du chapitre sur la socialisation et la construction des identités sociales, du programme de Première, et porte sur le format de l'évaluation sommative réalisée en classe par écrit. Sont impliquées deux classes du même lycée ${ }^{2}$, ayant le même professeur de SES (coauteure de cet article). Elles diffèrent peu par le niveau des élèves en début d'année, mais beaucoup par le climat de travail en classe, tel qu'il est perçu par l'enseignante. La première (notée $A, 23$ élèves) est globalement beaucoup plus attentive et participative que la seconde (notée B, 23 élèves, dont 4 absents à ce devoir proposant un support BD). L'écart dans les résultats entre les deux classes en SES s'est creusé au fil du temps. La moyenne de la classe A pour le trimestre concerné (le second) en SES est de 13,3/20 et celle de la classe B de 12,1/20 ; les moyennes respectives sur l'année pour les devoirs sur table sont de 11 pour la classe A et 10,5 pour la classe B. C'est la différence d'attitude des élèves qui a fondé le choix de réaliser le devoir en binôme dans la classe $\mathrm{A}$, sur $1 \mathrm{~h} 30$ mais individuellement dans la classe B, sur 1h50. Même s'il est relativement exceptionnel de proposer de réaliser un devoir sur table en binôme, en général, au lycée, l'enseignante de SES concernée, utilise fréquemment des dispositifs de pédagogie coopérative.

3 Alors qu'une des règles fondamentales du contrat didactique repose sur le fait que les élèves soient évalués sur des savoirs et savoir-faire qui leur ont été préalablement enseignés (Cohen, 1987; Biggs, 1996; Belec, 2016), les élèves ont découvert que leur support d'évaluation se présentait sous forme de BD le jour du devoir, et ne se sont vus donner aucune consigne spécifique pour en guider la lecture. En soi, ce fait constitue à la fois une rupture avec le contrat didactique (un format inhabituel), avec le principe d'alignement pédagogique (c'était la première fois qu'ils étaient confrontés à un exercice de ce type), et avec la mission de gestion didactique, qui aurait dû amener l'enseignante à les accompagner davantage dans l'appréhension de ce nouveau support. Globalement, la pratique étudiée est donc assez problématique. Des nuances doivent toutefois être apportées. Le support BD a été utilisé préalablement, même si aucun enseignement 
explicite n'a été réalisé sur les codes de lecture. Les élèves avaient déjà répondu en classe à des questions d'analyse sociologiques sur des strips de quelques cases ${ }^{3}$. La BD à analyser pour le devoir comportait quant à elle 7 planches. Un vrai changement d'échelle a donc eu lieu lors du passage à l'évaluation.

Il s'agissait d'un récit biographique extrait d'un album de Pénélope Bagieu (Les Culottées, t. 2, Gallimard, 2017) qui narre l'histoire de Sonita Alizadeh, une jeune afghane dont la famille fuit le régime des Talibans. Réfugiée en Iran dans un centre pour enfants, elle s'émancipe à l'adolescence en devenant une rappeuse et dénonce sur Youtube le mariage forcé des jeunes filles. Sa notoriété et sa pugnacité pour obtenir un passeport lui ouvrent les portes d'une scolarisation aux Etats-Unis, où elle poursuit son combat féministe. Le sujet demandait explicitement aux élèves de décrire d'un point de vue sociologique la vie de Sonita en utilisant de façon pertinente les concepts abordés en cours. Les notions attendues étaient les suivantes: socialisation primaire, socialisation secondaire, socialisation différentielle de classe, socialisation différentielle de genre, instances de socialisation (travail, famille, école, médias, groupe de pairs, associations ou clubs), socialisation anticipatrice, groupe de référence, groupe d'appartenance.

Il convient de préciser que la démarche d'analyse biographique consistant à décrire dans les termes de l'analyse sociologique une trajectoire de vie est relativement classique parmi les savoir-faire travaillés en classe de $\mathrm{SES}^{4}$. Les compétences disciplinaires mobilisées correspondaient bien à un exercice habituel, aligné avec les activités réalisées antérieurement en classe. Craignant que l'introduction du support BD n'entraîne une rupture de contrat didactique, l'enseignante a exhorté les élèves à prendre l'exercice au sérieux, même s'il s'appuyait sur un support original. Il a été rappelé que le devoir était noté et comptait dans la moyenne.

Comme il était demandé de définir chaque notion lors de leur première utilisation, certains élèves ont demandé s'ils pouvaient séparer cette partie définitionnelle du reste du devoir, car ils peinaient à l'intégrer dans le fil de leur analyse, ce qui a été accepté. Les copies recueillies sont donc de deux formats. Le premier, majoritaire, se présente en une seule partie, décrivant les principales étapes de la vie de Sonita qui peuvent être expliquées par les notions du cours, dont la définition est donnée directement lorsqu'elles sont appliquées à son cas. Le second est en deux parties: un lexique des notions sociologiques employées, puis une explicitation de la vie de Sonita mobilisant ces notions.

7 Le barème de notation, expliqué aux élèves au début du devoir, tient compte 1) de la perspicacité de la description pour embrasser les éléments essentiels qui façonnent l'identité sociale de la protagoniste au cours de sa vie (nationalité, religion, langue, genre, activité, etc) ; 2) de la capacité à appliquer les notions sociologiques, appréhendée en trois volets, à savoir utiliser le terme adéquat, le définir correctement, et en fournir un exemple tirée de la vie de Sonita (cf. corrigé en annexe 1). La correction a été réalisée en classe lors du cours qui a suivi le devoir, avant que les élèves ne se voient rendre leur copie notée.

\section{Un support nécessitant des compétences spécifiques de lecture}

8 L'esthétique générale de ce récit en sept planches correspond au style minimaliste d'un courant qui a émergé dans les années 90 , appelé «la nouvelle bande dessinée ${ }^{5}$. La difficulté de lecture résidait surtout dans l'impression première de densité textuelle et l'effet de miniature des vignettes, enserrées dans une composition en gaufrier qui ne 
permettait pas toujours de distinguer nettement les charnières du récit. Cette esthétique intellectualisante, qui est en rupture avec l'attractivité de l'image, dans les lectures BD traditionnelles des 7-14 ans, supposait pour le moins un temps d'acculturation et d'accompagnement spécifique, ce dont les élèves n'ont pas disposé.

Les sept planches se présentent sous une forme fixe. Elles sont composées d'un gaufrier de trois bandes horizontales, avec des cases verticales de même largeur, de manière à former un parfait quadrillage. La narration n'est pas pilotée par l'image, mais par des récitatifs en lettres capitales, qui occupent la moitié de la surface de chaque vignette et sont toujours placés en haut de celle-ci, avec parfois un ajout inférieur. Ce choix de composition et de mise en page crée immédiatement l'effet visuel d'une forte densité textuelle. Afin d'aérer la composition, plusieurs vignettes de la planche sont détourées, selon des distributions variées (en croix, en L ou en triangle), en fonction du contenu expressif. Cependant l'effet de concentration textuelle demeure, qui plus est dans un espace vignettal resserré et étroit. Les bulles sont très rares, et la plupart du temps employées pour des propos très courts, à deux exceptions près (qui vont au-delà de dix mots). L'écriture des paroles est en cursive, ce qui permet de distinguer ces dernières des récitatifs en lettres capitales. C'est parfois le seul signe distinctif pour les repérer, car les propos des personnages apparaissent très souvent sans phylactère. L'ensemble du dispositif donne l'impression d'un récit illustré séquentiel, plus que d'une bande dessinée qui exploiterait la variété des possibilités expressives de l'image. Du reste tous les personnages sont représentés de face ou de trois-quarts, en pied ou en plan rapproché. Il n'y a pas de profondeur de champ, les décors sont absents ou réduits au minimum. Il n'y a que trois occurrences de gros plan, une seule occurrence de plan d'ensemble et une seule vue en plongée. Le récit suit un ordre strictement chronologique. Les ellipses narratives sont des ellipses de moment à moment ou de scène à scène ${ }^{6}$. Sonita est représentée pratiquement à chaque case, de telle sorte qu'il semble commode pour le lecteur de suivre l'avancée de ce récit linéaire. Mais le caractère minimaliste de la narration peut brouiller le repérage des démarcations spatiale et temporelle dans les pérégrinations de la protagoniste. Sur le plan culturel, la BD suppose des connaissances sur le régime des talibans, la charia, et la proximité géographique entre l'Afghanistan et l'Iran.

\section{Parier sur l'effet facilitant du support BD}

Comment expliquer la mise en place d'une situation de lecture qui peut apparaître si évidemment problématique au regard de la théorie didactique? Présenté ainsi a posteriori, il paraît évident qu'il aurait été opportun de prévoir dans la séquence didactique un travail d'explicitation des codes de lecture du support BD. Cependant, à la décharge de l'enseignante, dans la pratique, le recours à une diversité de supports (BD, photo, vidéo, affiches, etc) sans problématisation explicite des codes sémiotiques associés est relativement courant en classe de SES. La plupart des ressources enseignantes, telles que les manuels, encouragent cette multiplication des supports. Les programmes ne mentionnent des savoir-faire de lecture spécifiques à propos de ceux qui mobilisent des compétences mathématiques.

11 L'enseignante a parié sur un effet «facilitant» de la BD, perçue comme motivante pour ses élèves. Il lui a semblé possible, sous cette forme, de les faire travailler sur un récit biographique un peu plus long que ce qu'elle leur aurait proposé sous forme de texte, en anticipant que ce format leur paraîtrait moins rébarbatif. 
12 Ce pari renvoie à la question du plaisir et, plus largement, des émotions attachées à la tâche, dans les situations d'apprentissage. La recherche internationale en éducation souligne en effet l'importance des aspects socio-émotionnels dans les processus d'enseignement-apprentissage (e. g. Baker, Järvelä, Andriessen, 2013). Ainsi, on reconnaît désormais aux émotions, perceptions indissociablement cognitives et physiologiques, caractérisées par une intensité (plus ou moins forte) et une valence (plus ou moins agréables), un rôle fondamental dans l'engagement et la réalisation d'une activité didactique (Baker, Andriessen, Järvelä, 2013, p. 14-15). Le plaisir pris dans un exercice peut ainsi être associé à une meilleure performance (e.g. Beal et al., 2003)

\section{Retour sur expérience : une pratique authentique aux effets contrastés}

13 Étant donné l'absence d'accompagnement des élèves vis-à-vis du support BD, on aurait pu s'attendre à ce que les résultats démontrent des difficultés à réaliser la tâche donnée. Or les notes moyennes, dans les deux classes, sont supérieures à celles obtenues pour l'ensemble des devoirs sur table de l'année : 14,2 pour la classe A contre une moyenne de 11 ; et 11,9 pour la classe $B$ contre une moyenne de 10,5 . Pour la classe $A$, la moyenne à ce devoir est également supérieure à la moyenne du trimestre (le second), qui est de 13,3/20 ; elle est à peine inférieure pour la classe $B$ (moyenne du second trimestre : $12,1 / 20^{7}$. Par ailleurs, les hiérarchies habituelles ont été parfois bousculées, certains élèves ayant habituellement de bons résultats se trouvant ici moins performants que d'autres qui ont souvent plus de difficultés. Comment expliquer ces changements?

\section{Méthodologie et questions de recherche}

14 C'est a posteriori que s'est posée la question de mener un travail de recherche à partir de cette expérience, pour démêler l'éventuelle présence d'un «effet BD », et trouver des pistes d'amélioration de cette pratique évaluative. À cette fin, nous avons recueilli deux types de matériaux : les copies des élèves ont été photocopiées, et un questionnaire libre a été passé aux élèves, après que le devoir a été corrigé en classe, et qu'ils ont eu connaissance de leur note. Ce questionnaire, passé en fin de cours sur un temps assez limité (15 minutes) comportait sept questions (cf annexe 2).

Les questions 2 et 3 visaient à cerner le profil des lecteurs, pour mesurer leur acculturation à la BD. Les questions 5 et 6 visaient à savoir si la lecture-compréhension de cette $\mathrm{BD}$ avait été perçue ou non comme facile. Les questions 1 et 4 visaient à comparer les représentations avant et après la lecture. La question 7 visait à savoir si ce support avait été perçu comme facilitant ou pas pour l'analyse. Certains élèves ayant fait le devoir n'ont pas répondu au questionnaire, soit qu'ils étaient absents, soit qu'ils ne souhaitaient pas le faire. Au total, nous avons recueilli les réponses de 18 élèves de la classe A et de 16 élèves de la classe B. En croisant les réponses des intéressés avec l'analyse de leur copie et leur note moyenne au devoir de fin de séquence, nous avons exploré les quatre questions de recherche suivantes :

- -La perception de difficulté ou de facilité de la tâche est-elle associée à une pratique plus ou moins développée de la lecture personnelle de BD, et, en particulier de BD de ce type ?

- Est-elle associée à des émotions spécifiques liées à la tâche? 
- Cette perception se traduit-elle concrètement, dans les copies des élèves, par l'absence ou la présence d'incompréhensions manifestes de l'histoire?

- Ces perceptions sont-elles cohérentes avec la note obtenue?

\section{Des résultats globaux contrastés}

\section{Déclaratif : niveau de difficulté ressenti et émotions liées au support}

$16 \mathrm{Au}$ regard des réponses au questionnaire, la grande tendance est l'absence de lecture personnelle de BD dans l'échantillon des deux classes de $2^{\text {nde }}$ : seulement 5 élèves (sur 34) déclarent continuer à en lire ; 10 élèves déclarent avoir cessé d'en lire depuis plusieurs années; les autres ne mentionnent aucune lecture de BD. Il est à noter que les élèves lecteurs de BD déclarés (présent ou passé) sont deux fois plus nombreux dans l'échantillon de la classe A que dans celui de la classe B (10 contre 5).

17 Les réactions premières des élèves devant la découverte du support BD sont toutes positives dans la classe A : ce choix est jugé intéressant (1 occurrence), différent, nouveau et original (6 occurrences), stimulant (3 occurrences), agréable ou sympa (2 occurrences), surprenant [dans le bon sens] ( 2 occurrences), potentiellement facilitant ( 1 occurrence). Les réactions sont plus contrastées dans la classe B : 6 réactions positives sans réserve, 2 réactions positives avec des réserves sur l'apparence de facilité, 6 réactions de surprise sans plus de précision, 4 impressions de bizarre[rie], 1 réaction d'incompréhension. Cependant tous les élèves de la classe B ayant répondu au questionnaire, sauf un, expriment un sentiment positif ou neutre lors de la lecture. Celle-ci est jugée facile (5 occurrences), plaisante (7 occurrences), ou normalement compréhensible (4 occurrences), en dépit du sentiment de longueur (5 occurrences). Seul un élève de la classe B exprime un sentiment uniquement négatif de longueur.

18 Au final, tous les élèves de la classe A, sauf un, ont trouvé que le support BD facilitait le travail d'analyse. Le rôle facilitant de l'image pour la compréhension est explicitement mentionné à deux reprises. Dans la classe $\mathrm{B}$, les résultats sont plus contrastés : 8 élèves sur 16 ayant répondu au questionnaire expriment au moins un aspect négatif dans leur lecture de la $\mathrm{BD}$, autre que celui de la longueur : 6 trouvent que ce support est plus difficile qu'un support textuel habituel ; 4 déclarent avoir découvert, lors de la correction, des éléments du récit qu'ils n'avaient pas compris.

\section{Analyse des copies : stratégies de résolution et erreurs de compréhension}

19 L'analyse de l'ensemble des copies des deux classes révèle trois modalités de traitement du sujet. Un traitement ascendant, qui consiste à évoquer chronologiquement la diégèse, pour retrouver les concepts de l'analyse sociologique. Un traitement descendant, qui consiste à partir des concepts pour les exemplifier par des éléments de l'histoire. Et un traitement dissocié, qui consiste à définir préalablement les concepts, avant un traitement de type ascendant.

Toutes les copies recueillies sauf deux présentent des erreurs de compréhension en lecture. Nous avons regroupé les erreurs en grandes catégories: contresens, extrapolation, généralisation hâtive, erreur sur l'identité d'un personnage, erreur de chronologie, erreurs liées au cadre spatial, erreur d'imputation causale, inférence erronée sur la motivation, ellipses narratives préjudiciables à la compréhension. Toutes les erreurs recensées ont été catégorisées dans le tableau disponible en annexe 5. Une copie 
de niveau faible présente également de grosses difficultés à recueillir des informations, l'élève affirmant à plusieurs reprises que le document ne mentionne pas certains éléments qui y figurent pourtant. Une bonne part de ces erreurs repose sur des inférences inadéquates, des ellipses spatio-temporelles, la description limitée du contexte, et certaines relations causales éludées. Ces erreurs sont tributaires du mode de lecture adopté (ascendant ou descendant selon que l'on parte de la diégèse ou des concepts sociologiques pour analyser le récit), mais aussi sans doute du médium BD lui-même. Ainsi, certaines des affirmations erronées des élèves peuvent s'expliquer par la primauté du recours à l'image sur la lecture du texte.

21 Ces résultats globaux contrastés rendent impossible d'établir un effet global du médium BD utilisé. Pour mieux comprendre ce que ce medium a pu produire de favorable ou de défavorable pour certains élèves, nous avons choisi de concentrer l'analyse sur 11 cas issus de la classe B : 5 élèves déclarant avoir éprouvé une plus grande difficulté avec ce support (groupe 1), et 6 élèves considérant que le support BD a été facilitant (groupe 2). Le choix d'avoir limité le panel d'étude de cas à cette classe s'explique par le caractère individuel du travail réalisé, par la nature contrastée des réponses, et par les résultats moyens obtenus. Nous avons ensuite comparé les notes obtenues avec la moyenne des intéressés aux devoirs surveillés de fin de séquence.

\section{Etudes de cas : multiples combinaisons entre perception et réussite de la tâche}

Les études de cas menées sur les groupes 1 et 2 donnent à voir que la perception de la tâche comme plus ou moins facile ou plaisante ne coöncide pas nécessairement avec le fait de réussir objectivement l'exercice, qu'il s'agisse d'avoir une bonne note ou de ne pas commettre d'erreurs de lecture. Le tableau 1 recense l'ensemble des configurations relevées pour ces onze cas ${ }^{8}$.

Tableau . Combinaisons de la perception du support BD et de la réussite de la tâche pour les 11 études de cas menées.

\begin{tabular}{|c|c|c|c|c|c|c|}
\hline & & & \multicolumn{4}{|c|}{ Perception recueillie par questionnaire } \\
\hline & & & \multicolumn{2}{|c|}{$\begin{array}{l}\text { Support BD plus } \\
\text { difficile }\end{array}$} & \multicolumn{2}{|c|}{$\begin{array}{l}\text { Support BD plus } \\
\text { facile }\end{array}$} \\
\hline & & & $\begin{array}{l}\text { Emotions } \\
\text { positives }\end{array}$ & $\begin{array}{l}\text { Emotions } \\
\text { négatives }\end{array}$ & $\begin{array}{l}\text { Emotions } \\
\text { positives }\end{array}$ & $\begin{array}{l}\text { Emotions } \\
\text { négatives }\end{array}$ \\
\hline \multirow{4}{*}{$\begin{array}{l}\text { Qualité de la copie } \\
\text { par rapport aux } \\
\text { objectifs } \\
\text { disciplinaires (note) } \\
\text { et à ra } \\
\text { compréhension de la } \\
\text { BD (lecture) }\end{array}$} & \multirow{2}{*}{$\begin{array}{l}\text { Note au } \\
\text { moins } 1 \text { point } \\
\text { supérieure à } \\
\text { la moyenne } \\
\text { de l'élève aux } \\
\text { DS }\end{array}$} & $\begin{array}{l}\text { Peu } \\
\text { d'erreurs } \\
\text { de lecture }\end{array}$ & Eliès & & $\begin{array}{l}\text { Géraldine } \\
\text { Franck }\end{array}$ & \\
\hline & & $\begin{array}{l}\text { Beaucoup } \\
\text { d'erreurs }\end{array}$ & & & Lili & \\
\hline & $\begin{array}{lr}\text { Note } & \text { proche } \\
\text { de } & \text { la }\end{array}$ & $\begin{array}{l}\text { Peu } \\
\text { d'erreurs }\end{array}$ & Elora & Serge & & \\
\hline & $\begin{array}{l}\text { moyenne de } \\
\text { l'élève aux DS }\end{array}$ & & & & & \\
\hline
\end{tabular}




\begin{tabular}{|c|c|c|c|c|c|}
\hline & $\begin{array}{l}\text { Beaucoup } \\
\text { d'erreurs }\end{array}$ & & Nathan & & \\
\hline \multirow{2}{*}{$\begin{array}{l}\text { Note au } \\
\text { moins } 1 \text { point } \\
\text { inférieure à la } \\
\text { moyenne de } \\
\text { l'élève aux DS }\end{array}$} & $\begin{array}{l}\text { Peu } \\
\text { d'erreurs }\end{array}$ & & & $\begin{array}{l}\text { Vincent } \\
\text { Maude }\end{array}$ & Julien \\
\hline & $\begin{array}{l}\text { Beaucoup } \\
\text { d'erreurs }\end{array}$ & Marie & & & \\
\hline
\end{tabular}

Les questions posées sur la difficulté perçue portaient d'une part sur le support BD luimême en comparaison à des documents plus classiques. Les émotions déclarées vis-à-vis du support BD incluent à la fois l'a priori à la découverte du document et l'impression laissée par l'expérience (cf. 2.1). Il convient de noter que lorsque l'élève n'a pas explicité de ressenti vis-à-vis du support, mais a par ailleurs exprimé une difficulté, il a été mis par défaut dans la catégorie "ressenti négatif ", l'expression de la difficulté renvoyant plutôt à un affect déplaisant. De plus, les niveaux de notes sont considérés de façon relative et non absolue : par exemple une «bonne note » correspond à une performance d'au moins 1 point supérieure à la moyenne des devoirs sur table de l'intéressé (voir le tableau cidessus).

\section{Elèves exprimant une plus grande difficulté avec le support BD}

Cinq élèves de la classe $\mathrm{B}$ ont déclaré que le support BD utilisé pour le devoir a constitué une difficulté (annexe 3). Leurs principales erreurs de compréhension du récit sont consignées dans l'annexe 5 .

\section{Nathan : beaucoup d'erreurs mais une note moyenne}

Nathan a obtenu la note de 6,75. Il affirme ne pas lire du tout de BD, avoir été surpris par le choix de ce support, avoir trouvé la lecture longue et plus difficile que pour un document classique. En revanche il pense avoir tout compris de l'histoire. L'analyse de sa copie montre une lecture ascendante, qui part du résumé de l'histoire pour tenter de reconstruire des concepts sociologiques. Or l'attention première à la diégèse révèle des problèmes de compréhension: on note des ellipses de temps et d'enchaînements de causalité, avec une absence de contextualisation précise sur le cadre, le lieu, l'âge et le statut des personnages. Une lecture purement factuelle tend à évacuer les enjeux et banaliser l'action: "C'est alors que pendant qu'elle travaille elle entend la radio ». Une telle phrase évacue le contexte du centre de réfugié pour enfants et l'impossibilité pour la protagoniste de trouver du travail en dehors. On note aussi une extrapolation: «Et quelque temps plus tard on la retrouve dans un cabaret maquillée et non plus vêtue d'un voile ». Il s'agit d'une erreur induite par la présence du mot «sundance » dans le décor, alors qu'il s'agit d'un festival américain du film indépendant. Le récitatif dit que le documentaire fait le tour du monde des festivals, et que Sonita y est invitée à témoigner, sans préciser explicitement qu'il s'agit de festivals de films. L'inférence pragmatique erronée provient aussi du fait que dans cette vignette l'héroïne porte une casquette américaine de rap. Nathan interprète donc la scène avec les connaissances culturelles qui sont les siennes. Quant au maquillage de Sonita, il s'agit en réalité d'une coloration du visage, rendu grisée 
par la photocopie en noir et blanc. Dans son commentaire, le professeur, à titre d'encouragement, note que le résumé est plutôt satisfaisant, mais qu'il y a un manque d'analyse sociologique. Tout laisse à penser tout de même que la lecture de la BD a posé des problèmes de compréhension.

\section{Elora : plaisir, peu d'erreurs et une note moyenne.}

Elora a obtenu la note de 12,5. Elle ne lit pas de BD, a fortiori de ce type. En découvrant le sujet, elle a d'abord pensé que le support serait facilitant. Elle a trouvé la lecture plaisante, mais longue. Après la correction, elle ne voit pas d'élément du récit qu'elle n'aurait pas préalablement compris. Au final, elle pense qu'il a été plus difficile de travailler avec la BD qu'avec un document classique. Son approche du récit est descendante. Elora maitrise bien les concepts sociologiques qu'elle prend le temps de définir, mais elle peine à les illustrer pleinement par l'exposé détaillé de la situation. Elle revient fréquemment à la définition générale. Il n'y a pas dans sa copie d'erreur de compréhension du récit vraiment significative. La note obtenue est malgré tout de 0,8 point inférieure à la moyenne de ses notes en devoir surveillé, même si elle reste dans la fourchette haute. Il est possible que l'absence de familiarisation avec la lecture de BD ait eu une influence sur la tendance de l'élève à rester dans la généralisation. En tout cas la BD ne semble pas l'avoir aidée à franchir le cap de l'exemplification.

\section{Serge : perception négative mais peu d'erreurs et une note moyenne.}

Serge a obtenu la note de 12,75. Il dit avoir trouvé le support BD plus difficile, et avoir ressenti une difficulté liée à la longueur. Sa réaction première a été la surprise. Il lit beaucoup de bandes dessinées, mais pas du tout de ce style-là. Après la correction, il n'identifie pas d'élément du récit qu'il n'aurait pas compris lors de la première lecture. De fait, sa copie, qui repose sur une approche dissociée, ne présente aucune erreur de compréhension de la diégèse. Parallèlement la plupart des notions sociologiques attendues sont en place. La note obtenue est inférieure à sa moyenne du trimestre $(13,4)$, mais légèrement supérieure à sa moyenne aux devoirs sur table tout au long de l'année $(12,6)$.

\section{Eliès : BD plaisante, peu d'erreurs et une note supérieure à sa moyenne}

Eliès a obtenu la note de 8,75 . Il affirme ne plus du tout lire de BD et n'avoir pas compris au départ pourquoi le devoir portait sur un tel support. Il a trouvé la lecture plaisante, mais longue, et il a constaté lors de la correction qu'il n'avait pas compris certains éléments de l'histoire. Au final il a trouvé ce type de devoir "plus difficile car non guidé ». Ce n'est donc pas seulement le choix de la BD qui est en cause, mais aussi le caractère global de la consigne, qui constituait une nouveauté. L'analyse de sa copie montre une lecture descendante, qui part des concepts sociologiques, pour retrouver dans la diégèse les éléments qui les illustrent. Cette approche non linéaire favorise les ellipses temporelles et causales. Ainsi, l'entrée par la notion d'instance de socialisation fait que l'élève passe sans transition de la famille au centre de réfugiés. De même la notion de groupe de pairs le conduit à passer sans transition du centre de réfugiés à l'école aux USA. Le devoir est ainsi marqué par une très forte décontextualisation. Toute la trame narrative de l'émancipation par le rap et la rébellion se trouve éludée. Eliès note par ailleurs qu'il ne trouve pas les éléments correspondant à la socialisation secondaire. 
La question est de savoir si c'est la difficulté de la lecture, qui l'empêche d'exemplifier la notion, ou si cette dernière est d'emblée mal maîtrisée. De façon corollaire, est-ce la difficulté de lecture, qui a entraîné ce mode d'approche descendant, ou bien ce dernier tend-il à complexifier la reconstitution de la diégèse et occulter des aspects du récit? Nous penchons pour cette seconde explication. La consigne globale a influé sur sa décision d'adopter un traitement descendant. Et cette approche non linéaire l'a pénalisé. La note obtenue est tout de même de 1,55 points supérieure à la note moyenne de ses autres devoirs surveillés. Le caractère plaisant de cette BD semble avoir eu un effet positif en dépit de la difficulté qu'a représenté pour lui la consigne globale.

\section{Marie : $\mathrm{BD}$ plaisante malgré de nombreuses erreurs et une note inférieure à sa moyenne}

Marie a obtenu la note de 9,5. Elle affirme ne pas lire du tout de BD. Alors qu'elle avait été agréablement surprise au départ, elle a trouvé la lecture plus difficile que pour un document ordinaire. Lors de la correction, elle a découvert des éléments de l'histoire qu'elle n'avait pas compris, la lecture lui a semblé longue, et elle garde au final un avis négatif sur ce choix. L'analyse de sa copie révèle une approche dissociée. Comme la consigne y invite, elle commence par définir les notions sociologiques vues en classe. Puis elle résume l'histoire en essayant de réintroduire les concepts. Si cette seconde approche limite les phénomènes d'ellipses, la copie en revanche révèle plusieurs problèmes de compréhension logique de l'action, comme le montre par exemple les assertions suivantes : «Encore une fois elle [Sonita] est exclue car elle n'est pas conforme à cette société». En réalité Sonita n'est pas exclue, c'est elle qui suit une trajectoire de rupture. " $L a$ réalisatrice veut que Sonita parte pour lancer sa carrière mais elle doit payer $2000 € »$. En réalité la réalisatrice ne veut pas que Sonita reparte se marier en Afghanistan, mais qu'elle continue le rap en Iran. C'est donc la réalisatrice qui paye la mère, afin que cette dernière reparte. Et c'est plus tard, que le succès de la vidéo permettra à Sonita de quitter le pays pour les USA. La réalisatrice ne veut donc pas d'emblée que Sonita parte pour les USA. L'élève condense et confond rétrospectivement plusieurs étapes distinctes, ce qui la conduit à formuler une motivation ou une causalité erronée. L'absence probable de relecture du récit, liée au sentiment éprouvé de longueur, explique sans doute ce type d'erreur et tend à mettre en cause le médium lui-même, pour une élève peu familiarisée à cette forme d'expression. La note obtenue est de 1,5 point inférieure à la moyenne de ses devoirs surveillés.

\section{Elèves qui expriment une facilitation de la tâche avec le support BD}

Sur les 16 élèves de la classe B qui ont répondu au questionnaire, 6 ont trouvé le support BD facilitant pour l'analyse, tandis qu'un élève a trouvé que c'était équivalent mais plus plaisant. Ces avis positifs ne préjugent pas du résultat ni des difficultés effectives de compréhension du récit. Les principales données déclaratives recueillies pour ces cas sont reproduites dans l'annexe 4 , et leurs principales erreurs de compréhension du récit dans l'annexe 5. 


\section{Maude : BD plaisante, peu d'erreurs, mais une note inférieure à sa moyenne}

31 Maude a obtenu la note de 11. Elle lit parfois des BD (de fantasy) et a déjà lu des BD biographiques de ce type. Elle a tout de suite trouvé que le support BD était une bonne idée, mais elle dit s'être méfiée d'emblée de l'impression de facilité qu'il peut donner. Elle a trouvé le sujet cohérent, la lecture agréable, n'a pas constaté après-coup de mécompréhension de sa part. Elle estime au final que ce support a été plus facilitant que certains autres documents. L'analyse de sa copie montre une approche descendante qui la conduit à faire des ellipses temporelles et causales. La copie n'évoque même pas la découverte du rap et la décision d'en faire. Cela semble aller de soi et demeure implicite. L'appropriation du médium BD ne semble pas être en cause. Pourtant, la note obtenue est nettement plus basse que sa moyenne trimestrielle (15) et que ses résultats habituels aux devoirs sur table (moyenne de 14,5 sur l'année). On peut se demander si, dans le cadre de ce nouvel exercice, ce n'est pas l'emploi d'une approche descendante qui a limité sa restitution du récit, malgré sa bonne maîtrise des concepts sociologiques.

\section{Julien : peu d'erreurs mais une perception négative et une note inférieure à sa moyenne}

32 Julien a obtenu une note de 6,75. Il ne lit pas de BD et a trouvé le choix du support «bizarre ». Mais surtout, il déclare ne pas voir le lien entre cette histoire et le cours sur la socialisation. Pour lui le sujet n'est pas « logique ». Par ailleurs il pense avoir tout compris et déclare que la BD a été facilitante, par rapport à la lecture d'un texte classique. Sa copie consiste exclusivement en un résumé de l'histoire, sans erreur de compréhension (à une légère inexactitude près), et surtout sans la moindre mention des notions vues en cours. La leçon n'a tout simplement pas été apprise. Sa note est de 1,35 points inférieure à sa moyenne en DS, elle-même artificiellement gonflée par de nombreuses absences.

\section{Lili : BD plaisante et note supérieure à sa moyenne malgré de nombreuses erreurs.}

Lili a obtenu la note de 13. Elle ne lit pas de BD, a fortiori de ce style, mais a été contente qu'un tel support soit proposé pour l'évaluation. Elle a trouvé le sujet cohérent avec le chapitre étudié et n'a pas constaté après correction de mécompréhension de l'histoire de sa part. Au final elle a trouvé le récit en BD plus facile pour l'application de concepts sociologiques. Sa copie propose une démarche de lecture ascendante, de la diégèse vers la conceptualisation, ce qui favorise une plus grande explicitation du contexte et limite les ellipses. Or plusieurs erreurs de compréhension demeurent présentes. Par exemple sur l'action : l'élève écrit qu'après la visite de son frère venu la chercher, Sonita rentre en Afghanistan auprès de sa mère qu'elle n'a pas vue depuis des années, alors que c'est sa mère qui vient à son tour la chercher. L'élève confond avec une étape ultérieure, lorsque Sonita rentre dans son pays chercher le passeport qui lui permettra de voyager aux EtatsUnis. Le résumé contient aussi des inexactitudes, par exemple le fait que le centre de réfugiés apprenne à Sonita à travailler pour se nourrir. Ces inexactitudes n'empêchent pas l'application satisfaisante des concepts de sociologie. La note obtenue est supérieure de 1,8 points à la moyenne de ses notes de devoir surveillé. 


\section{Franck : BD plaisante, peu d'erreurs et une note supérieure à sa moyenne}

\section{trouvé le sujet cohérent et n'estime pas avoir eu de problème de compréhension de} l'histoire, après la correction. Sa lecture s'est «bien passée » et il a trouvé que cette BD était un support facilitant pour l'analyse. Après avoir défini quelques notions du cours, Franck propose une lecture descendante. L'omission de plusieurs concepts clés le conduit à éluder plusieurs moments importants de la vie de Sonita. Le propos reste général et manque de précision contextuelle (éducation, religion, changement de pays, lutte, etc.). Cependant l'appropriation du médium BD ne semble pas en cause. La note obtenue est de 3 points supérieure à la moyenne de ses notes en devoir surveillé.

\section{Interprétation et discussion : y a-t-il un effet subjectivement facilitateur de la BD?}

\section{L'impossibilité d'objectiver un « effet BD »}

L'analyse des copies ne permet pas d'objectiver un « effet BD » en termes de qualité des productions des élèves : il n'y a pas de corrélation générale entre erreurs de lecture et réussite au devoir symbolisée par la note. Par exemple, on trouve beaucoup d'erreurs de compréhension dans la copie de Lili, mais celle-ci a obtenu une note supérieure à sa moyenne ; inversement, les copies de Vincent, Maude et Julien présentent peu d'erreurs de compréhension du récit, mais cela ne les a pas empêchés d'avoir une note inférieure à leur moyenne. En effet, la note dépend de trop de paramètres: effet du contexte didactique, de la consigne, du moment de l'année, du niveau de complexité du chapitre, etc. Enfin, la note obtenue reste « subjective ", comme toute évaluation quelle que soit la précision des critères employés.

De plus, il est assez naturel que la note ne soit pas un bon indicateur de l'effet BD car le barème de notation, en cohérence avec le contrat didactique de la classe de SES, était centré sur la maîtrise des notions disciplinaires et du travail d'analyse biographique. Ainsi, les compétences de lecture, si elles constituent des prérequis pour parfaitement réussir l'exercice, ne font pas elles-mêmes l'objet d'une évaluation explicite.

\section{Un support subjectivement facilitant?}

Contrairement au pari fait par l'enseignante, certains élèves ont bien souffert de la longueur du document (4 parmi les 5 exprimant une difficulté en lien avec la BD, cf. annexe 3). Cependant, beaucoup d'élèves expriment un a priori émotionnel positif vis-àvis du support BD et un goût a posteriori positif de cette expérience en devoir, parfois en dépit de la longueur, de la difficulté de la lecture (notamment dans les cas d'Eliès et d'Elora) et même de la note inférieure obtenue (en particulier dans les cas de Julien, Vincent, Elora et Maude). Sur les deux classes, seuls 3 élèves ont exprimé un a priori émotionnel négatif vis-à-vis du support BD (cf. tableau 1). Les 3 élèves déclarant ce médium de même difficulté ou plus difficile que les documents plus classiques le qualifient également de " plaisant » ou " agréable " ${ }^{9}$. On peut ainsi faire l'hypothèse que la $\mathrm{BD}$ a suscité un a priori émotionnel positif qui a aidé les élèves à s'investir dans la tâche. 
La BD semble globalement avoir suscité une certaine mise en confiance, un enrôlement plus facile, une certaine persistance dans la tâche (en dépit de la longueur), voire un certain plaisir.

Un biais important demeure, car ces appréciations ont été portées après connaissance de la note obtenue, ce qui a pu influencer a posteriori le ressenti ou la difficulté déclarée. Or seule une élève sur cinq de la classe $B$ ayant obtenu une note inférieure à sa moyenne (Marie) exprime un ressenti négatif au final (après avoir exprimé un a priori favorable).

Cela est d'autant plus remarquable que la grande tendance est l'absence de lecture personnelle de BD dans l'échantillon des deux classes: seulement 5 élèves (sur 34) déclarent continuer à en lire ; 9 élèves déclarent avoir arrêté d'en lire depuis plusieurs années; les autres ne mentionnent aucune lecture de BD. Si les élèves dans leur grande majorité supposent facile (à tort) la lecture d'un tel support, c'est sans doute parce qu'ils associent encore le médium aux lectures d'enfance.

40 A l'inverse l'expérience témoigne de vrais obstacles : tout d'abord, une surprise voire une déstabilisation première, liée à un sentiment de non-coïncidence entre le support et la discipline, mais plus encore des difficultés de lecture. Si le déclaratif des difficultés ne coïncide pas toujours avec la réalité des copies, globalement ceux qui se déclarent plus en difficulté avec la BD qu'avec un autre support, font quand même plus d'erreurs de compréhension. Ainsi il est significatif que dans la classe B, il y ait deux fois plus d'erreurs pour le groupe 1 (au moins 24), que pour le groupe 2 (11 erreurs dont 5 ellipses narratives ou généralisations). Si certaines erreurs sont imputables à un mode de lecture descendant et sélectif, en raison du caractère global de la consigne et des critères d'évaluation privilégiant la maîtrise des concepts sociologiques, on ne peut écarter le fait que caractère hybride, discontinu et elliptique des planches requière des compétences de lecture spécifiques, encore inégalement partagées.

\section{Conclusion}

41 En l'absence d'un travail d'acculturation préalable en classe, dans une pratique clairement désalignée, sur la forme, du reste de la séquence didactique, on observe un progrès général des notes au DS, dans les deux classes concernées. Au sein de chaque classe, les bons élèves réussissent indubitablement encore plus que les élèves en difficulté ou aux résultats moyens. S'il n'est pas possible d'objectiver un effet BD, ce qui l'emporte, c'est une perception émotionnelle globalement positive du choix BD, et ce, avant, pendant et après la lecture, même si cette perception est parfois non-corrélée aux difficultés effectives. Ainsi, l'effet négatif auquel un tel désalignement risquait d'aboutir semble avoir été limité par un effet subjectif, à savoir l'attachement d'émotions positives à ce médium, qui aurait facilité l'investissement des élèves dans une lecture relativement longue et une tâche difficile. Une telle interprétation plaide pour une complexification du triangle de la situation didactique (Brousseau, 1998), intégrant la spécificité du médium dans lequel le savoir est appréhendé, comme partie prenante importante de la dimension émotionnelle du rapport élèves-savoir.

42 Comment tirer parti de cet effet subjectif, pour favoriser les apprentissages ? Sans doute faudrait-il développer une acculturation au médium pour tous: il est certain qu'il a manqué dans cette séquence un temps d'enseignement sur les codes sémiotiques, ainsi qu'un entraînement spécifique en collectif sur un récit BD de même longueur. Plus 
largement, en s'inspirant des pratiques en didactique de la littérature, il serait sans doute possible de développer la tenue de carnets de lecture, en donnant à lire de façon cursive des récits qui mettent en image des concepts et des problématiques sociologiques, tels qu'en propose par exemple la collection BD « Sociorama », aux éditions Casterman.

\section{Annexes}

\section{Annexe 1 : Sujet et corrigé du devoir}

43 Sujet:

A l'aide de vos connaissances, vous fournirez une description sociologique de la vie de Sonita Alizadeh, rappeuse, expliquant les mécanismes qui façonnent son identité sociale au long de son parcours. Pour ce faire, vous utiliserez le vocabulaire spécifique appris en cours et définirez les notions-clefs associées avant de les appliquer à son cas.

\section{Corrigé :}

1 - Au cours de son enfance, Sonita acquiert un certain nombre de normes et de valeurs spécifiques à la société dans laquelle elle vit. Il s'agit de la socialisation primaire, qui définit son identité d'Afghane et musulmane. En tant que fille, elle intériorise des comportements féminins et subit les inégalités liées à cette condition dès le plus jeune âge. Les filles sont considérées comme des " poids financiers » et des objets à vendre pour le mariage. C'est la socialisation différentielle de genre. Sa socialisation primaire se fait principalement dans les interactions sociales au sein de sa famille, qui constitue quasiment l'unique espace social auquel elle a accès. Sonita intériorise, comme ses frères et sœurs, l'idée que le mariage ne se contracte pas par amour et que la femme doit obéissance à son époux, choisi pour elle par ses parents.

2 - A l'adolescence puis à l'âge adulte se construisent d'autres aspects de son identité sociale, qui entrent en contradiction avec sa socialisation d'origine. Il s'agit de la socialisation secondaire. Dès l'âge de 9 ans, elle évite de justesse un mariage arrangé, puis suit sa famille qui se réfugie en Iran. Son acculturation à la société iranienne s'inscrit à la fois en continuité de la culture afghane (religion, voile) et en rupture, dans un cadre affranchi du régime des talibans. Ses instances de socialisation secondaire sont la famille (réduite désormais à une sœur et sa fille), le centre pour enfants réfugiés, où elle apprend à lire et travaille, les médias culturels (la radio et la musique rap), le groupe des pairs (qui a une vision traditionnelle du mariage, mais va constituer son premier public et l'encourager), puis le milieu de la musique (studio de production). En commençant à rapper, Sonita suit une socialisation anticipatrice: elle intègre les pratiques et valeurs d'un groupe de référence (les rappeuses) afin de progressivement venir à en faire partie. 3 - Sa socialisation primaire a façonné durablement l'identité de Sonita. A 16 ans, elle se sent toujours sous l'autorité de son frère et de sa mère et doit négocier pour éviter un nouveau mariage arrangé.

4 - Dans le même temps, il y a du nouveau du côté d'une autre instance de socialisation : le travail. Grâce à sa rencontre avec une réalisatrice iranienne, elle obtient de l'argent pour un sursis de liberté et s'initie au cinéma. Son activité se situe à la frontière d'une autre instance de socialisation : elle devient une Youtubeuse, et acquiert les codes de ce nouveau travail rapidement. Ce succès lui ouvre les portes des Etats-Unis, d'une scolarisation et d'une carrière de rappeuse.

5 - L'émigration solitaire aux USA se situe en continuité et en rupture avec l'identité 
sociale de Sonita. La langue, la religion, la culture scolaire sont autres. Mais elle trouve une continuité dans le rap, l'écriture, et le projet de retourner en Afghanistan œuvrer à l'émancipation féminine. Son succès et son intégration dans une école privé américaine lui font expérimenter un décalage non seulement culturel mais aussi d'origine sociale. Elle qui a grandi en ayant faim, elle ne comprend pas par exemple ses camarades qui ne finissent pas leur assiette. C'est la prise de conscience d'une autre socialisation différentielle vécue dans son enfance et adolescence : une socialisation différentielle de classe.

\section{Annexe 2: Questionnaire passé aux élèves a posteriori}

1) Qu'avez-vous pensé du fait que le devoir propose une BD comme support de l'analyse sociologique? Quelle a été votre réaction première? Avez-vous été déconcertés, rebutés par cette idée, ou bien plutôt stimulés dans votre curiosité?

2) Lisez-vous des BD en dehors du temps scolaire? Si oui, lesquelles?

3) Avez-vous déjà lu des BD de ce style, avec un contenu biographique à la fois documenté et humoristique ? Si oui lesquelles?

4) À la fin du devoir, ce support vous a-t-il semblé cohérent avec le sujet, ou bien assez logique?

5) Après la correction, voyez-vous des éléments de la BD que vous n'aviez pas bien compris à la première lecture ? Lesquels?

6) La lecture de cette BD a-t-elle été facile, plaisante, longue, pénible, fastidieuse pour la compréhension?

7) Par rapport à l'étude d'un texte documentaire classique, cette BD a-t-elle facilité pour vous l'application des concepts sociologiques? Ou bien la forme particulière de cette BD vous a-t-elle rendu l'exercice plus difficile?

\section{Annexe 3 : Difficultés, sentiments et pratiques de lecture déclarées} des sondés du groupe 1

\begin{tabular}{|l|l|l|l|l|l|l|l|}
\hline Elève & Classe & $\begin{array}{l}\text { Note } \\
\mathrm{du} \\
\mathrm{DS}\end{array}$ & $\begin{array}{l}\text { Moyenne } \\
\mathrm{du} \\
\text { trimestre }\end{array}$ & $\begin{array}{l}\text { Moyenne } \\
\text { aux DS }\end{array}$ & $\begin{array}{l}\text { Pratique } \\
\text { de lecture } \\
\text { de la BD }\end{array}$ & $\begin{array}{l}\text { Difficulté } \\
\text { exprimée }\end{array}$ & $\begin{array}{l}\text { Emotions } \\
\text { déclarées }\end{array}$ \\
\hline
\end{tabular}




\begin{tabular}{|c|c|c|c|c|c|c|c|}
\hline Marie & B & 9,5 & 13,5 & 11 & Non & $\begin{array}{l}\text { Plus difficile de } \\
\text { travailler sur les } \\
\text { BD. } \\
\text { Après } \\
\text { correction : il y a } \\
\text { des choses que } \\
\text { j'avais pas } \\
\text { comprise sur le } \\
\text { moment } \\
\text { La lecture était } \\
\text { longue mais } \\
\text { simple } \\
\text { La BD est plus } \\
\text { difficile au final }\end{array}$ & $\begin{array}{l}\text { Première } \\
\text { impression : c'est } \\
\text { bien ca change }\end{array}$ \\
\hline Eliès & B & 8,75 & 7,5 & 7,2 & $\begin{array}{l}\text { Non, } \\
\text { plus } \\
\text { maintenant }\end{array}$ & $\begin{array}{l}\text { Incompréhension } \\
\text { Malgré les [...] } \\
\text { corrections il n'y } \\
\text { a des points non } \\
\text { compris } \\
\text { Plus difficile car } \\
\text { non guidé }\end{array}$ & $\begin{array}{l}\text { Surprise et } \\
\text { incompréhension } \\
\text { Plaisante à la } \\
\text { lecture mais pas } \\
\text { logique au début } \\
\text { Plaisante }\end{array}$ \\
\hline Nathan & B & 6,75 & 10,5 & 5,9 & Non & $\begin{array}{l}\text { [La lecture] a été } \\
\text { longue. } \\
\text { J'ai trouvé cela } \\
\text { plus difficile que } \\
\text { les docs habituels }\end{array}$ & $\begin{array}{l}\text { Un peu bizarre } \\
\text { d'avoir un BD à } \\
\text { analyser pour } \\
\text { faire un contrôle }\end{array}$ \\
\hline Elora & B & 12,5 & 13 & 13,3 & Non & $\begin{array}{l}\text { la lecture a été } \\
\text { plaisante mais } \\
\text { assez longue } \\
\text { J'ai trouvé plus } \\
\text { difficile de } \\
\text { travailler sur la } \\
\text { BD que sur les } \\
\text { documents. }\end{array}$ & $\begin{array}{l}\text { Première } \\
\text { réaction: c'est } \\
\text { bien et simple } \\
\text { Dernière } \\
\text { impression: } \\
\text { [cela] m'a semblé } \\
\text { assez logique } \\
\text { la lecture a été } \\
\text { plaisante }\end{array}$ \\
\hline Serge & B & 12,75 & 13,4 & 12,6 & $\begin{array}{l}\text { Oui plein, } \\
\text { mais pas de } \\
\text { ce style-là» }\end{array}$ & $\begin{array}{l}\text { La lecture a été } \\
\text { longue } \\
\text { Plus difficile de } \\
\text { travailler sur la } \\
\text { BD }\end{array}$ & $\begin{array}{l}\text { Première } \\
\text { réaction : surpris } \\
\text { Dernière } \\
\text { impression : } \\
\text { assez } \\
\text { compréhensible }\end{array}$ \\
\hline
\end{tabular}


Annexe 3 : Difficultés, sentiments déclarés et pratiques de lecture des sondés du groupe 2

\begin{tabular}{|c|c|c|c|c|c|c|c|}
\hline Elève & Classe & $\begin{array}{l}\text { Note } \\
\text { du } \\
\text { DS }\end{array}$ & $\begin{array}{l}\text { Moyenne } \\
\text { du } \\
\text { trimestre }\end{array}$ & $\begin{array}{l}\text { Moyenne } \\
\text { aux DS }\end{array}$ & $\begin{array}{l}\text { Pratique de } \\
\text { lecture de BD }\end{array}$ & $\begin{array}{l}\text { Facilité } \\
\text { exprimée }\end{array}$ & $\begin{array}{l}\text { Emotions } \\
\text { déclarées }\end{array}$ \\
\hline Franck & B & 9,5 & 10 & 6,4 & Non & $\begin{array}{l}\text { Par rapport aux } \\
\text { documents } \\
\text { habituels: plus } \\
\text { facile }\end{array}$ & $\begin{array}{l}\text { Première } \\
\text { impression : } \\
\text { Surpris } \\
\text { Dernière } \\
\text { impression : } \\
\text { logique } \\
\text { Lecture : bien } \\
\text { passée }\end{array}$ \\
\hline Julien & B & 6,75 & 9 & 8,1 (abs) & Non & $\begin{array}{l}\text { Lecture : } \\
\text { normale } \\
\text { Par rapport aux } \\
\text { documents } \\
\text { habituels: } \\
\text { lecture plus } \\
\text { facile }\end{array}$ & $\begin{array}{l}\text { Première } \\
\text { impression : } \\
\text { bizarre } \\
\text { Dernière } \\
\text { impression : } \\
\text { sujet pas } \\
\text { logique }\end{array}$ \\
\hline Lili & B & 13 & 14,2 & 11,2 & Non & $\begin{array}{l}\text { Lecture : facile } \\
\text { Par rapport aux } \\
\text { documents } \\
\text { habituels: plus } \\
\text { facile }\end{array}$ & $\begin{array}{l}\text { Première } \\
\text { impression : } \\
\text { j'étais } \\
\text { contente } \\
\text { Dernière } \\
\text { impression : } \\
\text { [cela] m'a } \\
\text { semblé assez } \\
\text { logique }\end{array}$ \\
\hline Vincent & B & 6,75 & 10,9 & 8,8 & Non & $\begin{array}{l}\text { La lecture était } \\
\text { compréhensible } \\
\text { car pas trop } \\
\text { longue et } \\
\text { plaisante } \\
\text { Non, pas plus } \\
\text { difficile de } \\
\text { travailler sur la } \\
\text { BD }\end{array}$ & $\begin{array}{l}\text { Première } \\
\text { impression : } \\
\text { ça m'a } \\
\text { surpris } \\
\text { Dernière } \\
\text { impression : } \\
\text { C'était en } \\
\text { lien avec le } \\
\text { chapitre } \\
\text { mais j'aurais } \\
\text { préféré des } \\
\text { questions } \\
\text { réponses }\end{array}$ \\
\hline
\end{tabular}




\begin{tabular}{|c|c|c|c|c|c|c|c|}
\hline Géraldine & B & 14,75 & 13,2 & 12,5 & Non & $\begin{array}{l}\text { Il y a des choses } \\
\text { que je n'avais } \\
\text { pas compris }\end{array}$ & $\begin{array}{l}\text { Première } \\
\text { impression : } \\
\text { Bien, original } \\
\text { Dernière } \\
\text { impression: } \\
\text { assez logique } \\
\text { avec le sujet. } \\
\text { J'avais tout } \\
\text { compris }\end{array}$ \\
\hline Maude & B & 11 & 15 & 14,5 & $\begin{array}{l}\text { Oui et parfois } \\
\text { des BD de ce } \\
\text { type } \\
\text { (biographique) }\end{array}$ & $\begin{array}{l}\text { la ça } \\
\\
\text { Lecture } \\
\text { agréable } \\
\text { C"est plus facile } \\
\text { que certains } \\
\text { docs mais co } \\
\text { dépend } \\
\text { lesquels. }\end{array}$ & $\begin{array}{l}\text { Première } \\
\text { impression : } \\
\text { le support } \\
\text { est une } \\
\text { bonne idée, } \\
\text { par contre ça } \\
\text { peut donner } \\
\text { une } \\
\text { impression } \\
\text { de facilité et } \\
\text { etre pris } \\
\text { moins au } \\
\text { sérieux } \\
\text { qu'un } \\
\text { contrôle } \\
\text { "classique". } \\
\text { Dernière } \\
\text { impression : } \\
\text { sujet logique } \\
\text { avec } \\
\text { chapitre le }\end{array}$ \\
\hline
\end{tabular}

\section{Annexe 4 : typologie des erreurs ou défauts de compréhension et d'interprétation}

\begin{tabular}{|l|l|l|l|}
\hline Elève(s) & Groupe & Classe & Extrait de copie (erreur soulignée) \\
\hline
\end{tabular}

\section{Contresens}

\begin{tabular}{|l|l|l|l|}
\hline Lia & & A & $\begin{array}{l}\text { (...) et attérit dans un centre pour enfants ou le travail est forcé et surtout pas } \\
\text { adapté à l'âge. }\end{array}$ \\
\hline Marie & 1 & B & Encore une fois elle est exclue car elle n'est pas conforme à cette société \\
\hline
\end{tabular}




\section{Extrapolations}

\begin{tabular}{|l|l|l|l|}
\hline Vincent & 2 & B & $\begin{array}{l}\text { Sonita qui vivait dans une famille pauvre où son père n'a pas eu de capital } \\
\text { culturel }\end{array}$ \\
\hline Géraldine & 2 & B & Lors des concerts elle devient un phénomène de mode \\
\hline Nathan & 1 & B & ( ?...) un faux mariage qui la ferait devenir une princesse \\
\hline Franck & 2 & B & $\begin{array}{l}\text { Cela va créer un choc chez elle et elle voudra à partir de ce moment-là } \\
\text { appartenir au même groupe que son chanteur préféré. }\end{array}$ \\
\hline Eliès & 1 & B & $\begin{array}{l}\text { On peut désormais dire que sa famille et sa religion ont donc été sont instances } \\
\text { sociales durant son enfance et plus tard donc adulte. }\end{array}$ \\
\hline
\end{tabular}

\section{Erreurs ou imprécisions sur l'identité d'un personnage}

\begin{tabular}{|l|l|l|l|}
\hline Maude & 2 & B & Sa mère a dû épouser un homme plus âgé (son oncle !) \\
\hline Julien & 2 & B & pour aller en Iran où elle restera avec sa sœur et une autre fille a Téhéran. \\
\hline Elora & 1 & B & Sonita s'est fait connaître par les médias grâce à une dame qui l'a filmée (...) \\
\hline
\end{tabular}

\section{Inférence d'action erronée}

\begin{tabular}{|l|l|l|l|}
\hline Nathan & 1 & B & maquillé et non plus vêtu d'un voile. \\
\hline Lili & 1 & B & On va lui apprendre à travailler pour gagner sa vie \\
\hline Géraldine & 2 & B & $\begin{array}{l}\text { Lorsqu'elle retourne en Afghanistan pour aller chercher son passeport, elle } \\
\text { remet son voile }\end{array}$ \\
\hline Lili & 1 & B & $\begin{array}{l}\text { Après qu'elle essaye de s'enfuir, elle atterrit dans un centre pour enfants } \\
\text { réfugiés }\end{array}$ \\
\hline
\end{tabular}

\section{Inférence de but erronée}

\begin{tabular}{|l|l|l|l|}
\hline Marie & 1 & $\begin{array}{l}\text { La réalisatrice veut que Sonia parte pour lancer sa carrière mais elle doit payer } \\
2000 €\end{array}$ \\
\hline
\end{tabular}




\section{Généralisation erronée ou hâtive}

\begin{tabular}{|l|l|l|l|}
\hline Elora & 1 & & $\begin{array}{l}\text { (...) la tradition de leur pays est d'être vendue à un homme et se marier le plus } \\
\text { tôt possible pour que la famille touche de l'argent dans ce cas l'argent revien au } \\
\text { frère (...) }\end{array}$ \\
\hline Eliès & 1 & B & $\begin{array}{l}\text { On voit que son instance sociale est la famille car tout au long de la bd, elle n'a } \\
\text { peur que du regard de sa famille }\end{array}$ \\
\hline Julien & 2 & B & $\begin{array}{l}\text { Sonita Alizadeh est une femme naît à Herat en Afghanistan, qui est contre la } \\
\text { charia et défend les femmes soumises à la charia. }\end{array}$ \\
\hline Vincent & 2 & B & $\begin{array}{l}\text { Tout au long de sa vie Sonita a subi des choses horribles (ex : forcée à se marier) } \\
\text { mais elle a toujours su les éviter (ex : en fuyant son pays) }\end{array}$ \\
\hline
\end{tabular}

\section{Erreurs liées à la chronologie des faits}

\begin{tabular}{|l|l|l|l|}
\hline Marie & 1 & B & $\begin{array}{l}\text { Elle arrive ensuite à destination où elle est prise en charge par un centre pour } \\
\text { enfants réfugiés (...) }\end{array}$ \\
\hline Eliès & 1 & B & $\begin{array}{l}\text { 2. On ne peut pas dire que leur groupe de pairs avec qui elle est dans ce centre est } \\
\text { une instance de socialisation car elle n'y reste peu longtemps (...) }\end{array}$ \\
\hline Lili & 1 & B & Elle rentre alors voir sa mère qu'elle n'a pas vu depuis des années (...) \\
\hline Nathan & 1 & B & Mais un jour son frère veut la vendre puisqu'il doit s'acheter une femme. \\
\hline
\end{tabular}

\section{Inférence de lieu erronée}

\begin{tabular}{|l|l|l|l|}
\hline Nathan & 1 & B & Et quelque temps plutard on la retrouve dans un cabaret \\
\hline Lili & 1 & B & $\begin{array}{l}\text { On peut parler de socialisation différentielle de classe parce qu'elle est dans un } \\
\text { restaurant, elle mange avec son amie et elle ne comprend pas pourquoi elle ne } \\
\text { finit pas son assiète. }\end{array}$ \\
\hline
\end{tabular}

\section{Erreurs liées à la causalité}

\begin{tabular}{|l|l|l|l|}
\hline Lili & 1 & B & Elle va y échapper [au mariage]. Après qu'elle essaye de s'en fuir, (...) \\
\hline Marie & 1 & B & $\begin{array}{l}\text { (...) elle veut se battre pour ses droits (...). Et pour ce fait elle commence à écrire du } \\
\text { rap (...) }\end{array}$ \\
\hline Marie & 1 & B & C'est d'ailleurs ce qui arrive lorsque son frère découvre ses projets. \\
\hline
\end{tabular}


Grâce à cette vidéo on lui propose d'intégrer une école privée aux Etats-Unis. Marie 1 B Avant de partir elle revoit sa famille qui lui tourne clairement le dos à cause de ses choix.

\section{Ellipses narratives (temps, cause, actions, cadre contextuel)}

\begin{tabular}{|l|l|l|l|}
\hline Nathan & 1 & B & «C'est alors que pendant qu'elle travaille elle entend la radio » \\
\hline Géraldine & 2 & B & «Puis elle part en Amérique » \\
\hline Franck & 2 & B & « Un jour elle va écouter une chanson d'une autre culture » \\
\hline Eliès & 1 & B & « Un peu plus tard on apprend qu'elle va aller dans une école privée » \\
\hline Maude & 2 & B & « Au USA... » \\
\hline Lia & & & $\begin{array}{l}\text { « Sonita se fait arrêter par les talibans et se retrouve dans un centre pour } \\
\text { enfants } \\
\text { (ici l'ellipse des actions intermédiaires peut faire croire que les talibans ont } \\
\text { placé Sonita dans le centre pour enfants, ce qui serait un contresens) }\end{array}$ \\
\hline
\end{tabular}

\section{Informations non trouvées}

\begin{tabular}{|l|l|l|l|}
\hline Eliès & 1 & B & $\begin{array}{l}\text { En bref le groupe de pairs ne lui a pas aidé à être la fille qu'elle est contrairement à } \\
\text { sa famille. }\end{array}$ \\
\hline Eliès & 1 & B & Pour sa socialisation secondaire on a pas d'informations (...) \\
\hline Eliès & 1 & B & On n'a aucune information sur cela. \\
\hline
\end{tabular}

\section{BIBLIOGRAPHIE}

Bagieu, P. (2017). Les Culottées, t. 2. Paris : Gallimard.

Baker, M., Andriessen, J., Järvelä, S. (2013). Introduction: visions of learning together. In M.

Baker, J. Andriessen, S. Järvelä (dir.), Affective Learning Together (pp. 1-30). Routledge.

Belec, C. (2016). L'alignement pédagogique des lectures. Correspondance, Vol 22 (4). La revue web sur la valorisation du français en milieu collégial. Québec : Centre collégial de développement de matériel didactique (CCDMD). 
Biggs, J. (1996). Enhancing teaching through constructive alignment. Higher Education, 32, 347-364.

Brousseau, G. (1998). Théorie des situations didactiques : didactiques des mathématiques 1970-1990. Grenoble : La pensée sauvage.

Cohen, S.A. (1987). Instructional alignment : Searching for a magic bullet, Educational Researcher 16(8), 16-20.

Evans, C. (2015). Profils de lecteurs, profils de lecture. In B. Berthou (dir), La Bande dessinée : quelle lecture, quelle culture? (pp. 17-44). Paris : éditions de la Bibliothèque publique d'information.

François C. (1992). Savoir et connaissance dans la perspective de la transposition didactique. Recherches en Didactique des Mathématiques, 12 (2.3), 221-270.

Fraisse d'olimpio S. (coord.), (2016). Manuel Sciences économiques et sociales 1ère ES. Paris : Belin.

Helsby, W. (1999). Comics in Education. The link between Visual and Verbal Literacies : how Readers read Comics. Thèse de doctorat. Faculty of Educational Studies. University of Southampton.

Lacelle, N. (2012). La déconstruction et la reconstruction des œuvres multimodales. Une expérience vécue en classe à partir des bandes dessinées Paul et Persépolis. In M. Lebrun, N. Lacelle \& J-F. Boutin, La Littératie médiatique multimodale. De nouvelles approches en lecture-écriture à l'école et hors de l'école (pp. 125-140). Québec: Presse de l'Université du Québec.

Lacelle, N. \& Lebrun, M. (2010). Pour une littératie médiatique multimodale dans un contexte d'inclusion au secondaire. Intercompreensão. Revista de Didáctica das Linguas, 9, 151-156.

Lacelle, N. \& Lebrun M. (2011). Développer la compétence à la lecture et à l'expression multimodales grâce à une didactique de la littératie médiatique critique. Aspects didactiques de la lecture, de la maternelle à l'université, AIRDF, collection didactique, 3, 205-224.

MacCloud, S. (2007). L'art invisible. Paris : Delcourt.

Missiou, M. (2012). Un médium à la croisée des théories éducatives : bande dessinée et enjeux d'enseignement. In N. Rouvière (dir), Bande dessinée et enseignement des humanités (pp. 79-98). Grenoble : Ellug.

Passard C., Perl P-O. (coord.), (2014). Manuel Sciences économiques et sociales 1ère ES. Paris : Bordas.

\section{NOTES}

1. La BPI (Bibliothèque publique d'information du Centre Pompidou) et la DEPS (Département des Etudes, de la Prospective et de la Statistique du Ministère de la Culture et de la Communication) ont piloté en 2011 une vaste enquête nationale sur la lecture de bande dessinée en France, auprès de 4580 personnes âgées de 11 ans ou plus, représentatives de la population française. Pour les résultats, voir Christophe Evans (2015). Il a notamment été mis en évidence un profil très lecteur de lecteurs de BD.

2. Il s'agit d'un lycée polyvalent et professionnel situé dans une petite ville de 7500 habitants à 20 $\mathrm{km}$ de Grenoble (Isère, 38). Le taux de réussite au baccalauréat pour le lycée général y était de $91 \%$ en 2017 , avec $53,7 \%$ des élèves se présentant à l'examen admis avec une mention, un résultat global légèrement en dessous de celui du département (taux de réussite : 93,5\%, part de mentions sur les candidats présents : 59,1\%).

3. Les documents BD utilisés lors de ce chapitre sont les suivants: deux vignettes tirées du manuel de 1ES de chez Belin, Edition 2016 (doc 1 p. 180 et doc 4 p. 181), et deux extraits de Riche Pourquoi pas toi de Marion Montaigne, Michel Pinçon et Monique Pinçon-Charlot (2013) de 
respectivement de 2 et 8 planches (pages 57-58 et 70-77). Ce dernier extrait, certes plus long, a été utilisé différemment, puisqu'il s'agit d'un questionnaire.

4. A titre d'exemple, le document 2 p. 178 du manuel utilisé dans ces deux classes (Bordas 2014), est un texte avec quelques éléments biographiques choisis, et la troisième et dernière question qu'il prévoit est la suivante: "Comment peut-on expliquer sociologiquement la trajectoire de Mohammed?». Pour des questions de propriété intellectuelle, nous ne pouvons malheureusement reproduire aucun de ces documents ici.

5. Le terme de "Nouvelle Bande-Dessinée" fut principalement employé au début des années 2000, pour décrire une nouvelle génération d'auteurs dans un livre d'entretien d'Hugues Dayez avec Blain, Dupuy/Berberian, Blutch, De Crécy, David B., Guibert, Rabaté et Sfar. La nouvelle bande dessinée, Bruxelles, Niffle, 2004.

6. Selon la typologie de Scott MacCloud (2007) : les ellipses de moment à moment représentent les différentes étapes d'une action et font très peu appel à l'ellipse; les ellipses de scène à scène enchainent des contenus éloignés dans le temps ou dans l'espace.

7. Tous les autres devoirs sur table de l'année correspondent à des entraînements aux exercices prévus en SES au baccalauréat, portant généralement sur plusieurs chapitres. C'est généralement à ce type d'exercice que les élèves ont les moins bonnes notes. La moyenne du trimestre prend également en compte des exercices oraux (interrogation, exposés en groupe), des TP en salle informatique (recherche documentaire et analyse synthétique guidées, généralement en binômes), ou des synthèses écrites courtes individuelles plus ou moins rédigées (parfois sous forme de plan détaillé ou de carte conceptuelle). Le devoir dont il est question ici, du point de vue de l'enseignante, constituait un exercice de difficulté intermédiaire entre le devoir sur table classique et la petite synthèse individuelle.

8. Afin de préserver la confidentialité des données, les prénoms des élèves ont été remplacés par des pseudonymes.

9. De même, dans la classe A, la seule personne qui exprime une difficulté spécifique liée au support affirme néanmoins que sa lecture a été « plaisante ».

\section{RÉSUMÉS}

Utiliser la BD en classe de SES au lycée peut-t-il avoir un effet facilitant pour les apprentissages? Cet article rend compte de l'utilisation de ce support pour l'évaluation finale d'une séquence sur la socialisation en $1^{\text {ère }}$ ES. L'analyse des copies de deux classes et d'un questionnaire donné a posteriori montrent qu'il n'est pas possible d'isoler un effet BD objectif global univoque. Procéder par étude de cas permet de mieux repérer des situations où l'image, par exemple, a plutôt facilité la lecture ou, au contraire, favorisé des raccourcis erronés. Cependant, la BD semble bénéficier globalement d'une perception émotionnelle positive même si ce ressenti n'est pas toujours corrélé aux difficultés effectives. Se dégage ainsi un effet subjectivement facilitant du medium, qui aurait limité le risque d'échec lié au désalignement pédagogique suscité par le manque d'acculturation préalable au médium.

Can the use of comic books in high school social sciences classroom facilitate the learning process? This paper reports on the use of this medium for the final written exam about socialization. Analyzing how two groups of students managed to do the task on the basis of both their written assessment and an a posteriori survey shows that it is difficult to identify a clear 
global effect of the comic support per se. Getting into qualitative case studies makes it possible to distinguish situations when the images facilitated student's understanding of the narrative from situations when such medium rather led to erroneous shortcuts. Nevertheless, the use of comic books seems to be globally associated with positive emotions, either or not correlated to reading mistakes. Such 'subjective facilitating effect' could explain why the students performed quite well in spite of the educational misalignement related to a lack of previous enculturation to the codes of comics.

\section{INDEX}

Keywords : comics, didactics of sociology, reading, assessment, emotions, educational alignment Mots-clés : bande dessinée, didactique de la sociologie, lecture, évaluation, émotions, alignement pédagogique

\section{AUTEURS}

\section{CLAIRE POLO}

Université Lumière Lyon 2, Institut des Sciences et Pratiques de l'Education et de la Formation ISPEF, Laboratoire Education Culture Politiques - ECP

\section{NICOLAS ROUVIÈRE}

Université Grenoble-Alpes 